\title{
Chitosan thermogels for local T lymphocyte delivery for cancer immunotherapy
}

\author{
Anne Monette ${ }^{1 *}$, Caroline Ceccaldi ${ }^{2}$, Sophie Lerouge ${ }^{2}$, Réjean Lapointe ${ }^{1}$ \\ From 30th Annual Meeting and Associated Programs of the Society for Immunotherapy of Cancer (SITC 2015) \\ National Harbor, MD, USA. 4-8 November 2015
}

\section{Background}

The success of systemic adoptive $\mathrm{T}$ cell transfer lies in the capacity of the antigen-experienced cytotoxic $\mathrm{T}$ lymphocytes to access and persist within the tumour microenvironment. The mimicking of tertiary lymphoid structures that promote a protective immune response against cancer can be achieved using an injectable biocompatible matrix that releases anti-tumour, proliferating $\mathrm{T}$ lymphocytes. Prime candidates for this application are liquid, chitosan-based, biocompatible thermogels which rapidly gelify at physiological temperatures. Therefore, we aimed to fine-tune an injectable chitosanbased thermogel formulation that would provide an environment permitting the three-dimensional proliferation and release of $\mathrm{T}$ lymphocytes whose activation state can be influenced by the surrounding conditions. We have developed a novel chitosan formulation that is both cytocompatible and injectable, and which has ideal mechanical properties and porosity for $\mathrm{T}$ cell encapsulation and growth. With such promising characteristics, we hypothesize that the injection of these $\mathrm{T}$ lymphocytes loaded hydrogels into the tumour microenvironment will provide a means for a continuous delivery of $\mathrm{T}$ lymphocytes towards the reprogramming of inflammation mechanisms and the reduction of tumour burden.

\section{Materials and methods}

Novel T cell cytocompatible chitosan thermogels were prepared using combinations of gelling agents. Their rheological properties, mechanical strengths, $\mathrm{pH}$, osmolality, and morphology were evaluated. Three formulations were selected for human $\mathrm{T}$ cell encapsulation. Biocompatibility was assessed using live/dead staining and fluorescent microscopy. Thermogel- and supernatant-derived $\mathrm{T}$ cells and tumour infiltrating lymphocytes were immunophenotyped over time using flow cytometry.

\section{Results and conclusions}

We have optimized thermogel formulations supporting the encapsulation of $\mathrm{T}$ lymphocytes in vitro. Use of flow cytometry and microscopy demonstrates which novel thermogel formulation is best suited for cell viability, proliferation, and escape over time, along with the maintenance of $\mathrm{T}$ lymphocytes cellular phenotype and an activation status that can be influenced by surrounding conditions. Our injectable three-dimensional T lymphocyte cultures may serve to complement current adoptive cell transfer immunotherapies.

\section{Authors' details \\ 'Université de Montréal / Centre de Recherche du Centre Hospitalier de I'Université de Montreal (CRCHUM), Montreal, PQ, Canada. école de technologie supérieure / Centre de Recherche du Centre Hospitalier de I'Université de Montreal (CRCHUM), Montreal, PQ, Canada.}

Published: 4 November 2015

doi:10.1186/2051-1426-3-S2-P36

Cite this article as: Monette et al:: Chitosan thermogels for local T

lymphocyte delivery for cancer immunotherapy. Journal for

ImmunoTherapy of Cancer 2015 3(Suppl 2):P36. 